\title{
The Design and Implementation of the Digital Image's Retrieval System
}

\author{
Hanfeng Li ${ }^{a}$, Mengyu Wang ${ }^{b}$ and Yan Liu ${ }^{\mathrm{c}, *}$ \\ School of Quartermaster Technology, Jilin University, Changchun 130062, China \\ a1038098064@qq.com, b742888092@qq.com, ${ }^{\text {c1 } 1403686446 @ q q . c o m}$
}

Keywords: Retrieval technology; Digital image; Retrieval system.

\begin{abstract}
With the rapid development of multimedia technology, image's retrieval has become a researching hotspot, as well as new growth point in the field and application of the current information retrieval. This paper takes the overview of the digital image's retrieval technology as the key point, by means of the explanation of retrieval system design based on the content, it discusses the realization of digital image's retrieval system.
\end{abstract}

\section{Introduction}

With the rapid development of multimedia technology, computer performance as well as the improved speed of network transmission, the way of information expression is gradually changed from single text to graphics, images, animation, video and other forms which are mainly multimedia information. Especially in many government departments, business organizations, researching institutions and hospitals, there are a lot of digital image data and digital video data. Therefore, how to effectively express, organize, manage, query and retrieve these massive visual data will become the problem that people urgently need to solve in the era of information age.

\section{The Overview of Digital Image's Retrieval Technology}

The advantages that digital image processing technology have: quantitative, precision, reproducibility, flexibility, large storage capacity. The development of computer hardware and software technology as well as the development of the special hardware used for image processing will improve the speed of processing constantly, which will catch up with or surpass the speed of human visual processing in some aspects.

The main research contents of digital image processing can be included: image transform, image compression, image enhancement and restoration, image segmentation, image description and feature extraction, image classification (recognition) and so on. Image retrieval will be involved in image transform, image enhancement and restoration, image description and feature extraction, image classification, etc.

The intelligent retrieval of digital image usually refers to content based image retrieval (CBIR), this technology can be extracted by the automatic extraction by machine including visual features: color, texture, shape, spatial relation, and so on, which can have similarity matching for images in the database as well as the querying sample images in the space of feature, then it can retrieve out the images that are similar to the sample.

\section{The Design of Content Based Image Retrieval System}

Content based image retrieval (CBIR) refers to the integrated software or hardware system with comprehensive functions including the image operation, processing, feedback and comparison, as well as management, maintenance and some other functions. Generally, it can include the general image features that are extracted by the process of digital image processing, which can be included image color, shape, texture and semantic characteristics, as well as the process of contrasting the similarities between the image features of the retrieval image and the image features in the database. The typical structure of CBIR system and the general process can be shown in Fig 1. 


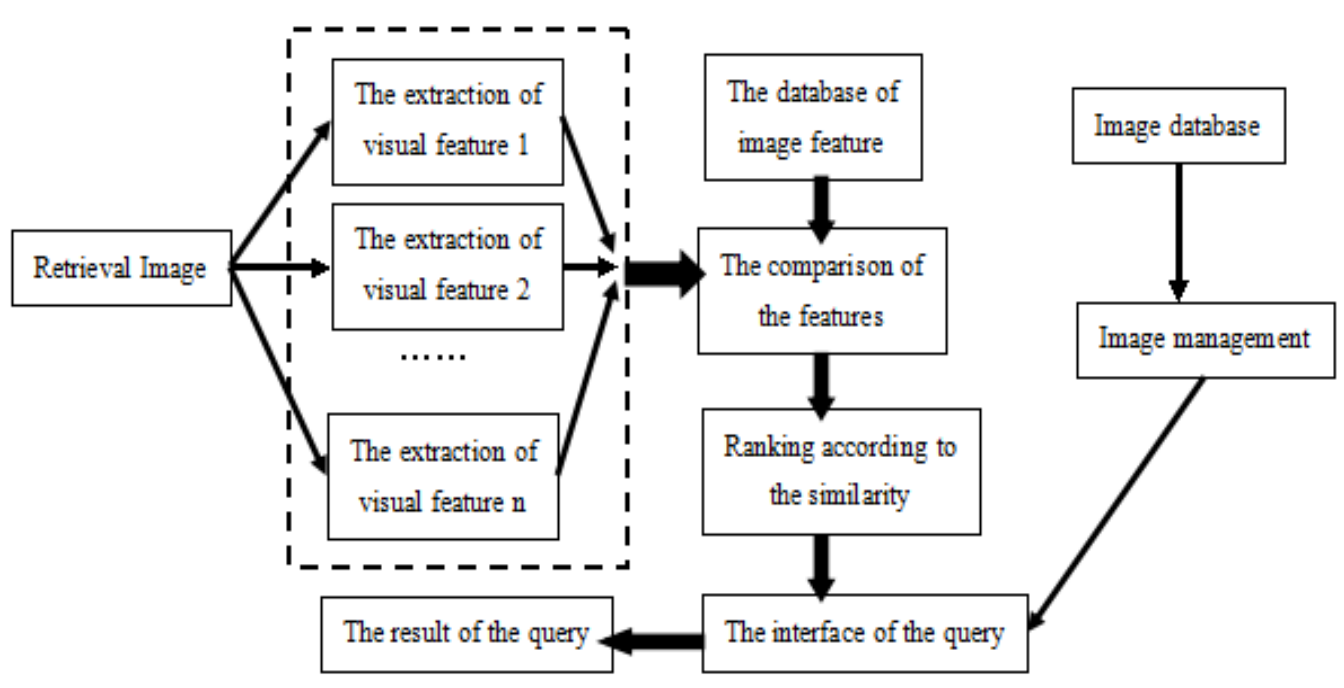

Fig 1. The flow chart of Typical structure of CBIR system

The process or the step of the content base image retrieval is usually included: first of all, the query retrieval image should be submitted by the user, and then the system will analyze it and extract the feature vector; then according to the corresponding similarity measurement criteria, matching them in image database; finally, according to the order of the similarity, it can output the retrieval result. Among them, the color, shape of the image, as well as the feature of texture has the relatively intuitive characteristics, while the semantic content has the relatively subjective and abstract characteristics. Although people prefer semantic query, it is not possible to have complete intelligent query for this mode so far, however, as for the direct image retrieval based on the visual features of the low level, people who are in query can quickly approach to the query results, which can greatly reduce the amount of workload.

\section{The Module Design of Digital Image's Retrieval System}

SttImage Retrieval is used in this paper to test the system, which also adopts database development of Visual C++6.0 and Oracle9i, using ADO technology to have access to the database. This system must run on the operating system of Microsoft Windows7, as for the hardware environment: Celeron2.66GCPU, as well as 512MB memory.

\section{The Management Module Design of Image Database}

The function of the management module of image database is to maintain the data of the database. The database of image feature of this system consists of three parts, namely, the original image, the data of features, image and other information. Imagedb table includes eight fields: ID, name of files, name, the length and width of image, the field of image, as well as the field of three characteristics: vector1, vector2, vector3.

\section{The Retrieval Module Design of Image Database}

The function of retrieval module is to provide various methods of inquiry for the user, so as to support the various types of queries work for users according to the specific requirements of different applications. This system adopts the example query, which can provide a wide range of inquiry, users can freely choose to express the feature, namely, who can adopt single feature such as BMCCM, SCH, CSSIM query, which also can combine them together to have comprehensive characteristic query. 


\section{The Realization of Digital Image's Retrieval System}

The Realization of Extracting Color Features. The color of the image has a variety of ways to express, the different description methods of color can represent the different color space correspondingly. It needs different color space in different applications. HSV model can express the color signals in three attributes: hue, saturation and value, this color model can be represented by the three-dimensional space coordinate system, namely, Munsell. The color mixing principle of RGB model is mixed with the additive method, that is to say all kinds of colors of light will be added and it will be brighter and brighter.

The Conversion Algorithm of RGB and HSV Color Model. In order to achieve it conveniently on the computer, it can be achieved by using mathematical formulas from the RGB color coordinate, the formula is as follows:

Defining the RGB color space with color value $(r, g, b)$, among them, $r, g, b \in[0,255]$, then, the value of h, s, v can be calculated in the transformed space, namely, HSV with the following formula:

$$
v^{\prime}=\max (r, g, b)
$$

$v=v^{\prime} / 255$

$s=\frac{v^{\prime}-\min (r, g, b)}{v^{\prime}}$

$h^{\prime}=\left\{\begin{array}{c}\left(5+b^{\prime}\right), \text { if } r=\max (r, g, b) \text { and } g=\min (r, g, b) \\ \left(1-g^{\prime}\right), \text { if } r=\max (r, g, b) \text { and } g \neq \min (r, g, b) \\ \left(1+r^{\prime}\right), \text { if } r=\max (r, g, b) \text { and } b=\min (r, g, b) \\ \left(3-b^{\prime}\right), \text { if } g=\max (r, g, b) \text { and } b \neq \min (r, g, b) \\ \left(3+g^{\prime}\right), \text { if } b=\max (r, g, b) \text { and } r=\min (r, g, b) \\ \left(5-r^{\prime}\right), \text { otherwise }\end{array}\right.$

$h=60 \times h^{\prime}$

$r^{\prime}, g^{\prime}, b^{\prime}$ is defined as

$r^{\prime}=\frac{v^{\prime}-r}{v^{\prime}-\min (r, g, b)}, g^{\prime}=\frac{v^{\prime}-g}{v^{\prime}-\min (r, g, b)}, b^{\prime}=\frac{v^{\prime}-b}{v^{\prime}-\min (r, g, b)}$

Here $r, g, b \in[0,255], h \in[0,360], s \in[0,1], v \in[0,1]$.

\section{The Realization of the Features of Color}

In the index, it can only store the main image's features of the color distribution, when it has feature matching, people can use these main color features to calculate out the similarity between the colors distributions of the image.

The experimental steps of the specific algorithm are as follows:

Step 1. Image preprocessing: firstly, image format can be converted into $256 * 256$; choose the HSV model to quantify, through color quantization, the three components of $\mathrm{H}, \mathrm{S}, \mathrm{V}$ can be distributed with different weights on a one-dimensional vector, then color component 8X3X3 can be got. them.

Step2. The image can be divided into blocks according to 3x3, so as to get 9 regions, and label

Step3. Calculating of the main characteristic vector of the color distribution in these 9 regions $\left(E_{i}, \sigma_{i}, S_{i}\right), 1 \leq i \leq 9$.

Step4. Calculating the degree of similarity between the images, the calculation formula is as follows:

$\left.D(Q, I)=\sum_{i} W_{i}\left|W_{E}\right| E_{I}^{Q}-E_{i}^{I}\left|+W_{\sigma}\right| \sigma_{i}^{Q}-\sigma_{i}^{I}\left|+W_{S}\right| S_{i}^{Q}-S_{i}^{I} \mid\right]$

$i \in[l, 9], W_{i}, 1 \leq i \leq 9$ can represent the weights of the visual importance in each region. 


\section{The Realization of Shape Feature Extraction}

As far as the image is concerned, shape is its only one feature that is important. At the same time, the concept of shape itself is very subjective, different people will give different descriptions.

The image's retrieval steps based on shape are as follows:

Step1. Extracting through the edge, getting the shape and contour of the object of the image.

Step2. Having seed filling, getting the targeted area surrounded by the contour line of the image.

Step3. Calculating the invariant moment of the targeted region, as well as the feature vector of constituting the shape of the image.

Step4. Having internal normalization to the feature vector of the shape, then the feature values can be stored in the image's feature database.

Step5. Having similarity matching. Using the euclidean distance of the two graphs' normalized eigenvectors as the similarity degree of two images. Assuming that the two graphs' normalized eigenvectors are $\left(h_{1}^{I}, h_{2}^{I}, \cdots, h_{7}^{i}\right)$ and $\left(h_{1}^{q}, h_{2}^{q}, \cdots h_{7}^{q}\right)$, then the function of euclidean distance is:

$$
S_{i m}(Q, I)=\sqrt{\sum_{i=1}^{n}\left(h_{i}^{q}-h_{i}^{I}\right)^{2}}
$$

Taking the smaller n (l-20), returning n (l-20) similar images.

\section{Conclusion}

Image's feature description as well as the similarity measurement is the core link of the image's retrieval system. The current capability to describe the low-level features of images are still far unable to cover the fundamental content of the image, the description of the spatial information is a hot issue in the field of image retrieval in recent years; in addition, the standardized testing platform needs to be strengthened. The standardized testing platform should include database, the defination of the correct result, as well as the system criterion.

\section{Reference}

[1]M. Flickner, H. Sawhney, W. Niblack, et al. 1995, Query by image and video content: the QBIC System. IEEE Computer. vol.28, pp23-32.

[2]J. Z. Wang, J. Li, G. Wiederhold. 2001, SIMPLIcity: Semantics—sensitive Integrated Matching for Picture Libraries. IEEE Transactions on Pattern Analysis and Machine Intelligence. Vol.23, pp9.

[3]J. R. Bach. 1996, The virage image search engine: An open framework for image management. Proc. SPIE: Storage and Retrieval for Still Image and Video Databases. IV 2670, pp76-87.

[4]Sclaroff S et al. 1997, ImageRover: a content--based image browser for the World--Wide Web. in Proceedings of IEEE Workshop on Content--Based Access of Image and Video Libraries, San Juan, Puerto Rico 1.23, pp2-9. 